\title{
Fibroblast growth factor 21 (FGF21) is increased in MDD and interacts with body mass index (BMI) to affect depression
} trajectory

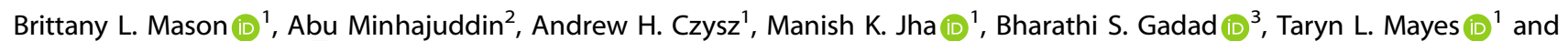 \\ Madhukar H. Trivedi (iD) ${ }^{\circledR}$
}

(c) The Author(s) 2022

Fibroblast growth factor 21 (FGF21) is a key regulator of metabolic function and nutrient preference. It also affects biological pathways associated with major depressive disorder (MDD), including corticotrophin-releasing hormone (CRH), leptin, and sympathetic activity. Lower levels of cerebrospinal fluid FGF21 have been associated with higher Beck Depression Inventory scores. FGF21 was examined as a metabolic marker that could be associated with MDD and evaluated as a biomarker of antidepressant treatment response in a large, randomized placebo-controlled trial in chronic, early-onset MDD participants. FGF21 levels at baseline and during treatment were determined for participants in the Establishing Moderators and Biosignatures of Antidepressant Response for Clinical Care (EMBARC) study. FGF21 was analyzed by ELISA in individuals with chronic, early-onset MDD (first major depressive episode before 30 years) compared to healthy control participants. Participants with MDD had higher levels of FGF21 compared to healthy controls (HCs), even after controlling for baseline age, sex, race, Hispanic ethnicity, BMI, and site $(\beta$-coefficient $=1.20, p<0.0001$, Cohen's $d=0.60)$. FGF21 did not change over time nor differ between treatment groups. Interestingly though, those with normal BMI and lower FGF21 levels showed a reduction in depression severity over time compared to all other groups. In conclusion, depression is associated with higher levels of FGF21 compared to healthy controls and those with lower levels of FGF21 (25th percentile of the sample) in the context of normal-weight BMI seem to have improved depression severity over time.

Translational Psychiatry (2022)12:16; https://doi.org/10.1038/s41398-021-01679-y

\section{INTRODUCTION}

Obesity and associated metabolic dysfunction have been shown to significantly impact the biology, disease course, and treatment response of major depressive disorder (MDD) [1, 2], and yet the basic mechanisms underlying these associations are largely underrecognized. Understanding the specific biological mechanisms related to the development of depression and related outcomes are essential to target and refine treatment selection within the heterogeneity of depression.

Fibroblast growth factor 21 (FGF21) is a key hormonal regulator of metabolic function and nutrient preference. Its production can be induced in the liver by diverse metabolic stressors, including starvation and protein-deficient diets, as well as simple sugars and ethanol, and has been shown to act directly on the nervous system to suppress sweet and ethanol preference, increase thermogenesis, and improve insulin sensitivity [3, 4]. It is known to affect biological signaling cascades implicated in depression, including the corticotrophin-releasing hormone $(\mathrm{CRH})$, leptin, and the sympathetic nervous system pathways [4]. FGF21 is also associated with poor metabolic health, with serum FGF21 levels being positively correlated with adiposity, fasting insulin and triglycerides, and increased risk of metabolic syndrome associated with high FGF21 [5]. This increased risk in the context of higher adiposity may be related to FGF21 resistance, which has been demonstrated in obese mice having significantly reduced FGF21 signaling response in both liver and fat tissues following exogenous administration of FGF21 [6]. FGF21 was not associated with stress-induced increase in cortisol in humans 3 months following a period of chronic stress, but was positively correlated with self-reported ability to cope with the stress [7], suggested that FGF21 may also play a role in managing the consequences of psychological stress. There are fewer data to support how FGF21 may be related to psychiatric conditions that also involve psychological stress, such as MDD.

FGF21 has been previously examined in depression and bipolar disorder in small studies. Lower levels of FGF21 in the cerebrospinal fluid (CSF) was associated with higher scores on the Beck Depression Inventory in Chinese men, but not women, who were not diagnosed with a mood disorder [8]. Changes in FGF21 following valproate treatment for bipolar depression in bipolar II disorder were associated with Hamilton Depression Rating Scale (HDRS) scores, with individuals having increased FGF21 showing worse outcomes as measured by HDRS scores [9]. Given these associations, FGF21 was explored as a metabolic

\footnotetext{
'Department of Psychiatry, UT Southwestern Medical Center, Dallas, TX, USA. ${ }^{2}$ Department of Population and Data Sciences, UT Southwestern Medical Center, Dallas, TX, USA. ${ }^{3}$ Department of Psychiatry, Texas Tech University Health Sciences Center El Paso, Dallas, TX, USA. ${ }^{凶}$ email: Madhukar.trivedi@utsouthwestern.edu
} 
marker that could be associated with MDD and evaluated as a biomarker of treatment response in a large, randomized, placebocontrolled trial of sertraline in chronic, early-onset MDD participants. The following questions were examined:

1) Are there differences in FGF21 levels in those with MDD compared to Healthy Controls?

2) Among participants with MDD, do levels of FGF21 change over 8 weeks of treatment, and are there demographic, clinical, or treatment characteristics that influence changes in FGF21 over time?

3) Are changes in depression severity associated with baseline FGF21 in the context of body mass index (BMI)?

\section{PARTICIPANTS AND METHODS \\ Participants}

The Establishing Moderators and Biosignatures of Antidepressant Response for Clinical Care (EMBARC) study was a randomized, doubleblind, placebo-controlled trial of the serotonin selective reuptake inhibitor sertraline. Full rationale and design has been detailed previously [10]. The study enrolled 296 participants across four sites: the University of Texas Southwestern Medical Center in Dallas, TX; Columbia University in New York, NY; the University of Michigan in Ann Arbor, Ml; and Massachusetts General Hospital in Boston, MA. Enrolled MDD participants were 18-65 years old, fluent in English, and had chronic, early-onset MDD (first major depressive episode prior to age 30 years) as diagnosed by the Structural Clinical Interview for DSM-IV. MDD participants were excluded if they scored $<14$ on the Quick Inventory of Depressive Symptomatology-Self Report baseline, had any other primary mental disorder other than MDD, had a lifetime history of a psychotic or bipolar disorder, had a substance abuse disorder within 6 months (except for nicotine dependence), or had the presence of a medical condition that would compromise MDD-specific findings. Forty healthy control participants were also enrolled, who had no personal history of or first-degree relative with a mood disorder.

This study was approved by the Institutional Review Board at each institution and all participants signed informed consent. Enrollment was active from 2011 to 2015. The study was registered with ClincialTrials.gov (NCT01407094).

\section{Treatment and assessments}

Upon meeting all inclusion and no exclusion criteria, MDD participants were randomized to double-blind, 8-week course of either sertraline or placebo in stage 1 of the study. Randomization was in $1: 1$ ratio and stratified by site. Dosing, which was started at $50 \mathrm{mg}$ of sertraline or similar placebo, with potential weekly titration of $50 \mathrm{mg} /$ day up to $200 \mathrm{mg} /$ day of sertraline or similarly increased placebo, was based on clinical decision, guided by measurement-based care principles. At the end of stage 1, responders continued their respective active treatment or placebo. Nonresponders in the sertraline arm were switched to bupropion, whereas those in the placebo arm were switched to sertraline. Both MDD and healthy control subjects were followed for 16 weeks. Only data from stage 1 of the study were analyzed for this report.

A range of clinical characteristics were collected during the EMBARC study, including symptoms of depression, mania, anxiety, anger, and suicide, as well as physical and medical characteristics, such as pain, sexual functioning, adverse effects, etc. (see [10] for full details). Outcomes on severity of depressive symptoms and changes over time were evaluated using HDRS (HAMD-17 [11]), which was collected at Baseline and at postrandomization Weeks $1,2,3,4,6$, and 8 during the stage 1 of the study.

\section{Body mass index}

Participant's vital signs were obtained at each visit and BMI was calculated at each time point. In general, BMI was evaluated as a continuous measure. For categorical displays of $\mathrm{BMI}$, standard $\mathrm{BMI}$ categories of normal weight $\left(18.5-24.9 \mathrm{~kg} / \mathrm{m}^{2}\right)$, overweight $\left(25-29.9 \mathrm{~kg} / \mathrm{m}^{2}\right)$, obese $\left(30-34.9 \mathrm{~kg} / \mathrm{m}^{2}\right)$, and morbidly obese $\left(>35 \mathrm{~kg} / \mathrm{m}^{2}\right)$ were used.

\section{Blood collection and processing}

Venous blood samples were collected in EDTA tubes at Baseline for all participants and at Weeks 1 and 8 for MDD participants. The samples were then isolated for plasma at each site. Plasma aliquots were frozen and stored at $-80^{\circ} \mathrm{C}$ until frozen shipment to the Rutgers University Cell \& DNA Repository (RUCDR). The full set of plasma samples from all sites was received frozen from RUDCR and stored at $-80^{\circ} \mathrm{C}$ until analysis.

\section{Quantification of analyte}

FGF21 was analyzed using analyte-specific enzyme-linked immunoabsorbant assays (FGF21: BioVendor Czech Republic), with a required dilution step to ensure that values would fall within the limits of detection and were adjusted for dilution after assay as is the standard practice. The limit of detection was $7 \mathrm{pg} / \mathrm{mL}$ and the inter-assay variability was $n=6$; coefficient of variation $(\mathrm{CV})=3.3 \%$. Interplate controls were used to detect any batch effects. In general, FGF21 was evaluated as a continuous measure. Low and high categories of FGF21 were defined as the 25th percentile (low) and the 75th percentile (high) of the sample.

\section{Statistical analyses}

FGF21 concentrations were calculated as described and adjusted for the dilution factor. The control values were evaluated for batch effects by scatterplot and no batch effect was detected for this sample set. All values were $\log _{2}$-transformed for use in statistical comparisons. Some samples measured were below the lower limit of detection and could not be included in analyses.

Continuous data were summarized as mean $\pm S D$ or median and interquartile range. Categorical data were summarized as frequency and percentages.

Analyses of covariance (ANCOVA) was used to assess whether baseline level of $\log _{2}$-transformed FGF21 were different between the MDD and HC control samples. Baseline age, sex, race, Hispanic ethnicity, BMI, and study site were used as covariates. In the MDD sample, baseline levels of $\log _{2^{-}}$ transformed FGF21 were compared between sertraline and placebo groups using ANCOVA analyses with baseline levels of depression severity (as measured by HAMD-17), baseline age, sex, race, Hispanic ethnicity, BMI, and study sites as covariates.

To assess whether FGF21 levels changed during treatment in the MDD sample, a repeated-measures ANCOVA (repeated ANCOVA) analysis was used with $\log _{2}$-transformed FGF21 levels as outcome, time (Week 0 , Week 1 , and Week 8) as a within-subject factor, and randomization groups (sertraline vs. placebo) as a between-subject factor and the interaction between them. Baseline depression (HAMD-17) severity, baseline age, sex, race, Hispanic ethnicity, BMI, and study site were included in the model as covariates. If the Group $\times$ Time interaction effect was statistically significant, the two treatment groups were compared at Weeks 0,1 , and 8 .

To assess the effects of baseline FGF21 levels on the changes in depression severity in the MDD sample, a repeated ANCOVA analysis was used with HAMD-17 at Baseline and Weeks 1, 2, 3, 4, 6, and 8 as the outcome, time as the within-subject factor, and baseline FGF21 levels as the covariate of interest. Time by FGF21 interaction was also included in the model. Given the known differences of FGF21 levels across obesity levels [12], participant BMI, time by BMI interaction, as well as a three-way interaction between time, FGF21, and BMI were also included in the model. In addition, baseline age, sex, race, Hispanic ethnicity, treatment group, and study site were included in the ANCOVA model.

All analyses were done using SAS 9.4 (SAS, Inc., Cary, NC) and statistical significance was assessed at $p<0.05$. At this time, no adjustments were made for multiple comparison.

\section{RESULTS}

EMBARC enrolled 309 participants with MDD and 40 healthy controls across the four sites. Of these 309 participants with MDD, the first 10 participants with MDD were randomized to citalopram and 3 participants were ineligibly randomized. Thus, the modified intent-to-treat stage 1 EMBARC sample included 296 participants randomized to sertraline or placebo. Not all participants provided a blood sample at every blood collection visit and not all participants were retained through all of stage 1. Of these, 207 provided blood samples that were able to be evaluated for FGF21 (102 sertraline and 105 placebo). Among the $40 \mathrm{HC}, 32$ provided blood samples that were able to be evaluated FGF21. Thus, the modified intent-to-treat sample for this report includes 207 participants with MDD, who were randomized and $32 \mathrm{HC}$ participants. Among the MDD sample, 184 provided blood 

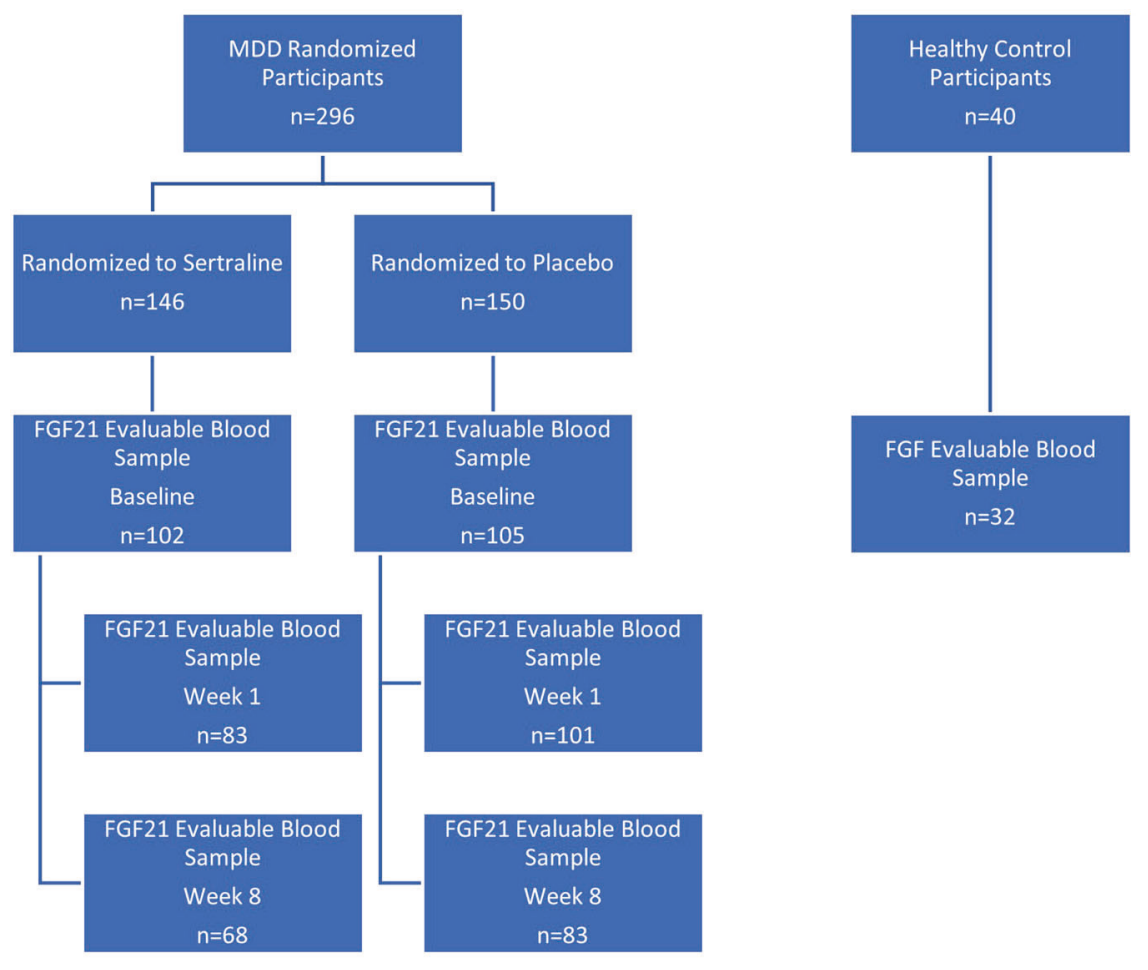

Fig. 1 Consort diagram.

\begin{tabular}{|c|c|c|c|}
\hline Variable & MDD $n=207$ & HC $n=32$ & $p$-Value \\
\hline Age, years, mean (SD) & $38.9(13.2)$ & $38.8(15.1)$ & 0.988 \\
\hline Sex, $n(\%)$ & & & 0.552 \\
\hline Female & $140(67.6)$ & $20(62.5)$ & \\
\hline Male & $67(32.4)$ & $12(37.5)$ & \\
\hline Race, $n(\%)$ & & & 0.710 \\
\hline White & $138(66.7)$ & $30(67.5)$ & \\
\hline African American & $39(18.8)$ & $8(25.0)$ & \\
\hline Other & $30(14.5)$ & $4(12.5)$ & \\
\hline Ethnicity & & & 0.082 \\
\hline Hispanic & $40(19.3)$ & $2(6.2)$ & \\
\hline Non-Hispanic & 167 (80.7) & $30(93.8)$ & \\
\hline Site & & & 0.005 \\
\hline Columbia & $63(30.4)$ & $9(28.1)$ & \\
\hline Massachusetts General & $16(7.7)$ & $9(28.1)$ & \\
\hline Michigan & $51(24.6)$ & 7 (21.9) & \\
\hline UTSW & $77(37.2)$ & 7 (21.9) & \\
\hline BMI, mean (SD), kg & $29.1(7.5)$ & $25.7(5.8)$ & 0.029 \\
\hline HAMD-17, mean (SD) & $18.5(4.5)$ & $0.6(0.8)$ & $<0.0001$ \\
\hline $\log _{2}(F G F 21)$ & $7.3(1.3)$ & $6.3(1.5)$ & $<0.0001$ \\
\hline
\end{tabular}

BMI values missing for $8 \mathrm{HC}$ and $22 \mathrm{MDD}$ patients.

samples that were able to be evaluated FGF21 at Week 1 (83 sertraline and 101 placebo) and 151 provided blood samples that were able to be evaluated for FGF21 at Week 8 (68 sertraline and 83 placebo) (Fig. 1). Of the samples analyzed for FGF21, 32 of these samples were below the lower limit of detection and not included in the analyses.
Table 2. $\log _{2}$ FGF21 levels over the course of treatment for sertraline vs. placebo.

\begin{tabular}{|llll|} 
& Sertraline & Placebo & Test statistic (p-value) \\
\hline Baseline & $7.3(1.3)$ & $7.4(1.2)$ & $0.9(0.390)$ \\
\hline Week 1 & $7.1(1.5)$ & $7.2(1.2)$ & $0.1(0.705)$ \\
\hline Week 8 & $7.2(1.5)$ & $7.2(1.4)$ & $0.2(0.788)$ \\
\hline
\end{tabular}

The demographic and clinical characteristics of the sample are presented in Table 1 . The majority of participants were female (67.6\% MDD and $62.5 \% \mathrm{HC}$ ), with a mean age around 38 years. Most were non-Hispanic and Caucasian. MDD participants had higher BMI $(p=0.029)$ and HAMD-17 scores $(p<0.0001)$. There were no other significant differences between the MDD and HC samples, and there were no statistical differences between the sertraline and placebo participants.

\section{Are there differences in FGF21 levels in those with MDD compared to Healthy Controls?}

Mean FGF21 level for MDD participants at Baseline was significantly higher compared to that for HC participants $(7.3 \pm$ $1.3 \mathrm{pg} / \mathrm{mL}$ vs. $6.3 \pm 1.5 \mathrm{pg} / \mathrm{mL} ; p<0.0001)$, even after adjusting for baseline age, sex, race, Hispanic ethnicity, BMI, and site ( $\beta$ coefficient $=1.20, p<0.0001$, Cohen's $d=0.60$ ).

\section{Among participants with MDD, do levels of FGF21 change over 8 weeks of treatment, and are there demographic, clinical, or treatment characteristics that influence changes in FGF21 over time?}

Table 2 provides the mean FGF21 levels at Baseline and Weeks 1 and 8 for MDD participants. Repeated ANCOVA (adjusted for baseline HAMD-17, baseline age, sex, race Hispanic ethnicity, BMI, and study site) revealed no changes in FGF21 levels over the 8 -week treatment phase (main effect of time $p=0.4411$ ) or between participants in sertraline and placebo groups (main effect 
Table 3. Change in FGF21 levels over 8 weeks by treatment group by Stage 1 treatment.

\begin{tabular}{lllrl|} 
Source & $\begin{array}{l}\text { Numerator } \\
\text { DF }\end{array}$ & $\begin{array}{l}\text { Denominator } \\
\text { DF }\end{array}$ & $\begin{array}{l}\boldsymbol{F} \text { - } \\
\text { value }\end{array}$ & $\begin{array}{l}\boldsymbol{p} \text { - } \\
\text { Value }\end{array}$ \\
\hline Time & 2 & 299 & 0.82 & 0.4411 \\
\hline $\begin{array}{l}\text { Treatment group } \\
\begin{array}{l}\text { Time } \times \\
\text { treatment group }\end{array}\end{array}$ & 1 & 187 & 0.07 & 0.7978 \\
\hline $\begin{array}{l}\text { HAMD-17 at } \\
\text { Baseline }\end{array}$ & 1 & 300 & 0.49 & 0.6116 \\
\hline $\begin{array}{l}\text { Age at evaluation } \\
\text { Sex }\end{array}$ & 1 & 187 & 0.76 & 0.3833 \\
\hline Race & 1 & 179 & 12.75 & 0.0005 \\
\hline Hispanic ethnicity & 1 & 187 & 0.29 & 0.5880 \\
\hline Site & 2 & 187 & 2.70 & 0.0698 \\
\hline BMI & 1 & 181 & 1.17 & 0.2800 \\
\hline & 1 & 198 & 1.11 & 0.3456 \\
\hline
\end{tabular}

of treatment group $p$-value $=0.7978)$. In addition, there was no significant time by treatment group interaction $(p=0.6116)$, suggesting that participants in the two treatment groups did not show differences in FGF21 changes over the 8 weeks. Other factors examined as covariates also did not impact change in FGF21 over 8 weeks, with the exception of baseline age, with older age being associated with greater change in FGF21 level (Table 3).

\section{Are changes in depression severity associated with baseline FGF21 in the context of BMI?}

A significant three-way interaction for FGF21, time based on repeated ANCOVA, and BMI was observed, suggesting that the effect of baseline FGF21 on changes in HAMD-17 over time depends on the BMI levels of participants $(F=3.52$, d.f. $=6,742$, $p=0.0019$ ). Specifically, among patients with normal BMI, those with low FGF21 (FGF21 $=95.6 \mathrm{pg} / \mathrm{mL}$ at baseline, 25th percentile) had significantly greater reduction in HAMD-17 score over time compared to participants with high FGF21 (FGF21 $=293.3 \mathrm{pg} / \mathrm{mL}$ at baseline, 75th percentile). However, there were no significant differences in HAMD-17 change over time based on low or high FGF21 in the other groups of participants who were overweight or obese. In addition, main effects of time, baseline HAMD total score, and site, as well as time $\times$ BMI and time $\times$ baseline FGF21 interactions were also associated with HAMD-17 scores. See Table 4 and Fig. 2 for details.

\section{DISCUSSION}

Using data from the EMBARC study, FGF21 was found to be higher in those with MDD compared to HC and FGF21 remained relatively stable over time and was not impacted by depression severity or by treatment. Importantly, when FGF21 levels and BMI were considered together, a significant three-way interaction was found. Participants with lower BMl, classified as normal weight, and lower FGF21 being in the 25th percentile of the sample had a greater decrease in depression severity as measured by the HAMD-17 compared to those with higher FGF21 being in the 75th percentile of the sample. There was no difference for participants with higher BMI, including those classified as overweight or obese, regardless of their levels of FGF21. These findings could indicate that elevation in FGF21 and elevated BMI may be biomarkers of treatment resistance.

The results from this study strengthen the evidence that increased FGF21 is associated with MDD and may have an impact on outcomes for certain individuals (i.e., those of normal weight). The finding that FGF21 was higher in individuals with MDD is
Table 4. Change in HAMD-17 scores over 8 weeks by levels of baseline FGF21 and BMI.

\begin{tabular}{lllcr|} 
Effect & Num DF & Den DF & $\boldsymbol{F}$-value & $\boldsymbol{p}$-Value \\
\hline Time & 5 & 762 & 5.95 & $<0.0001$ \\
\hline BMI & 1 & 166 & 1.03 & 0.3105 \\
\hline BMI $\times$ Time & 5 & 760 & 4.52 & 0.0005 \\
\hline FGF21 at Baseline & 1 & 169 & 2.28 & 0.1330 \\
\hline FGF21 $\times$ Time & 5 & 763 & 4.35 & 0.0007 \\
\hline BMI FGF21 $\times$ Time & 6 & 742 & 3.52 & 0.0019 \\
\hline HAMD-17 at Baseline & 1 & 164 & 36.73 & $<0.0001$ \\
\hline Age & 1 & 160 & 7.40 & 0.0072 \\
\hline Sex & 1 & 161 & 0.61 & 0.4345 \\
\hline Race & 2 & 163 & 0.17 & 0.8413 \\
\hline Hispanic ethnicity & 1 & 162 & 2.18 & 0.1417 \\
\hline Site & 3 & 164 & 5.98 & 0.0007 \\
\hline Treatment group & 1 & 162 & 0.67 & 0.4142
\end{tabular}

somewhat in contrast to previous research showing that lower levels of CSF FGF21 were associated with higher BDI scores in Chinese men, but not women, and without official diagnosis of depression [8]. This finding was from a small sample size and taken from the CSF and not plasma, which may affect this relationship even though FGF21 can cross the blood-brain barrier [13]. Furthermore, all participants in the study by Liu and colleagues had BDI scores less than 17, indicating no more than mild depressive symptoms.

Identification of the interaction between FGF21, BMI, and depression improvement over time suggests that FGF21 is important for people with MDD, who have a lower BMI, but for obese patients other mechanisms may be related to treatment outcomes. FGF21 acts on both peripheral and central targets [4], and has been shown to regulate preference for both sweet and alcohol, which may be dopamine-related [3, 14]. As dopamine signaling is implicated in mood regulation and reward perception [15], this could suggest that some aspect of FGF21 signaling is directly related to mood. However, given the numerous biological systems that are affected by FGF21, including activation of the sympathetic nervous system or indirect action on CRH [16], determining how FGF21 contributes to mood and the development of MDD will require further research. It is unclear whether the results suggest some effect contributing directly to the expression of depressed mood or instead suggest that FGF21 resistance in the system more broadly may be associated with a decreased ability to respond to treatment. The attenuated change in depression severity in those normal-weight participants with higher FGF21 does suggest that some aspect of FGF21 signaling is perpetuating depressive symptoms.

There are several limitations of this report. First, the sample size of healthy controls is relatively small and larger sample sizes are needed to confirm the findings. Second, the inclusion and exclusion criteria for the EMBARC study may limit the generalizability of these findings. Selection of FGF21 as a metabolic biomarker of interest may not be adequate to fully understand the interplay between metabolic biomarkers and depression symptoms, in particular given that weight likely plays an important role in metabolic markers, as evidenced by the results.

Importantly, these analyses highlight the interaction of BMI with the potential of a biomarker effect. The relationship between BMI and increased psychiatric symptoms, including MDD, is well known [17-19]; however, BMI is not frequently included as a variable by which treatment effects are evaluated. Simply controlling for this variability in the model may hide key biological differences in these responses. Given the different physiological 

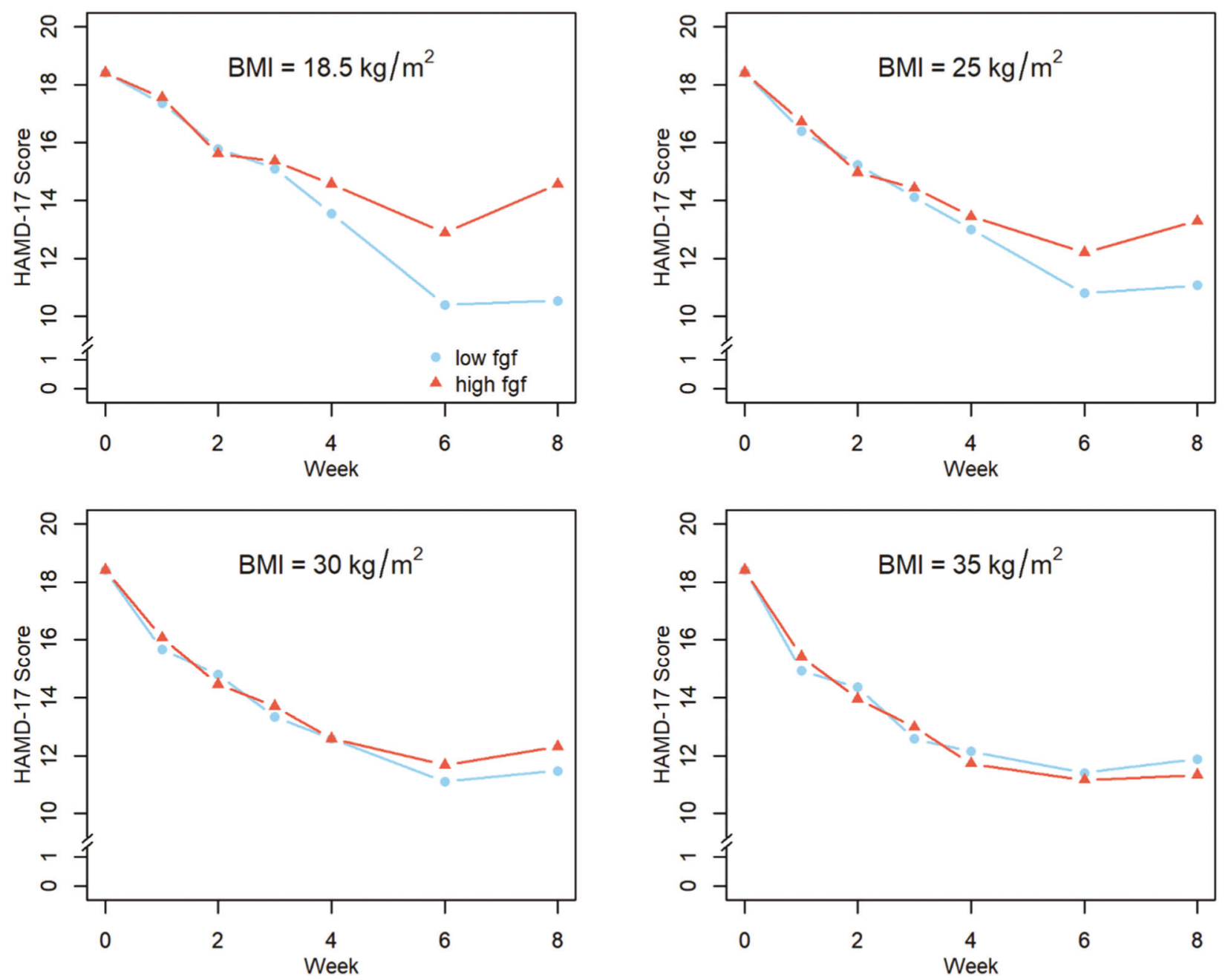

Fig. 2 Change in HAMD-17 score over 8 weeks for participants with low versus high FGF21. BMI of $18.5-24.9 \mathrm{~kg} / \mathrm{m} 2$ (normal weight), $25-29.9 \mathrm{~kg} / \mathrm{m} 2$ (overweight), 30-34.9 kg/m2 (obese I), and $35 \mathrm{~kg} / \mathrm{m} 2$ (obese II). Low and high FGF21 are defined as 25 th and 75 th percentiles, respectively.

states present in those with obesity, it is wise to consider that biological responses or treatment responses likely differ dependent on the metabolic state of the person. These analyses indicated that other factors may influence levels of FGF21 such differences between sexes or between racial and ethnic groups; however, larger studies have indicated that such interactions have not been significant and instead FGF21 was determined to be valuable as a biomarker for metabolic syndrome in multi-ethnic populations $[20,21]$. Thus, these findings from the EMBARC study support the association of FGF21 with MDD and provide further evidence that metabolic changes in MDD are worthy of further study as biomarkers. In addition, inclusion of BMI as a specific factor by which groups are stratified may further clarify how biological factors or biomarkers of interest may be differentially impacted. BMI could be a clinically useful marker, as it is easily obtained in clinical practice and could lead to better understanding of treatment response and non-response, particularly in the context of metabolic markers like FGF21.

\section{REFERENCES}

1. Hryhorczuk C, Sharma S, Fulton SE. Metabolic disturbances connecting obesity and depression. Front Neurosci. 2013;7:177-177.

2. Jha MK, Wakhlu S, Dronamraju N, Minhajuddin A, Greer TL, Trivedi MH. Validating pre-treatment body mass index as moderator of antidepressant treatment outcomes: findings from CO-MED trial. J Affect Disord. 2018;234:34-37.
3. Talukdar S, Owen BM, Song P, Hernandez G, Zhang Y, Zhou Y, et al. FGF21 regulates sweet and alcohol preference. Cell Metab. 2016;23:344-9.

4. BonDurant LD, Potthoff MJ. Fibroblast growth factor 21: a versatile regulator of metabolic homeostasis. Annu Rev Nutr. 2018;38:173-96.

5. Zhang X, Yeung D, Karpisek M, Stejskal D, Zhou ZG, Liu F, et al. Serum FGF21 levels are increased in obesity and are independently associated with the metabolic syndrome in humans. Diabetes. 2008;57:1246-53.

6. Fisher M, Chui PC, Antonellis PJ, Bina HA, Kharitonenkov A, Flier JS, et al. Obesity is a fibroblast growth factor 21 (FGF21)-resistant state. Diabetes. 2010;59:2781-9.

7. Jelenik T, Dille $M$, Müller-Lühlhoff $S$, Kabra DG, Zhou Z, Binsch $C$, et al. FGF21 regulates insulin sensitivity following long-term chronic stress. Mol Metab. 2018;16:126-38.

8. Liu Y, Wang M, Tan X, Wang X, Yang X, Xiao J, et al. Negative correlation between cerebrospinal fluid FGF21 levels and BDI scores in male Chinese subjects. Psychiatry Res. 2017;252:111-3.

9. Chang HH, Chen PS, Cheng YW, Wang TY, Yang YK, Lu RB. FGF21 is associated with metabolic effects and treatment response in depressed bipolar II disorder patients treated with valproate. Int J Neuropsychopharmacol. 2018;21:319-24.

10. Trivedi MH, McGrath PJ, Fava M, Parsey RV, Kurian BT, Phillips ML, et al. Establishing moderators and biosignatures of antidepressant response in clinical care (EMBARC): rationale and design. J Psychiatr Res. 2016;78:11-23.

11. Hamilton M. Development of a rating scale for primary depressive illness. Br J Soc Clin Psychol. 1967;6:278-96.

12. Tan BK, Hallschmid M, Adya R, Kern W, Lehnert H, Randeva HS. Fibroblast growth factor 21 (FGF21) in human cerebrospinal fluid: relationship with plasma FGF21 and body adiposity. Diabetes. 2011;60:2758-62. 
13. Hsuchou H, Pan W, Kastin AJ. The fasting polypeptide FGF21 can enter brain from blood. Peptides. 2007;28:2382-6.

14. von Holstein-Rathlou S, BonDurant LD, Peltekian L, Naber MC, Yin TC, Claflin KE, et al. FGF21 mediates endocrine control of simple sugar intake and sweet taste preference by the liver. Cell Metab. 2016;23:335-43.

15. Nusslock R, Alloy LB. Reward processing and mood-related symptoms: an RDoC and translational neuroscience perspective. J Affect Disord. 2017;216:3-16.

16. Kliewer SA, Mangelsdorf DJ. A dozen years of discovery: insights into the physiology and pharmacology of FGF21. Cell Metab. 2019;29:246-53.

17. Opel N, Thalamuthu A, Milaneschi Y, Grotegerd D, Flint C, Leenings R, et al. Brain structural abnormalities in obesity: relation to age, genetic risk, and common psychiatric disorders: Evidence through univariate and multivariate megaanalysis including 6420 participants from the ENIGMA MDD working group. Mol Psychiatry. 2020. https://doi.org/10.1038/s41380-020-0774-9.

18. Rao WW, Zong QQ, Zhang JW, An FR, Jackson T, Ungvari GS, et al. Obesity increases the risk of depression in children and adolescents: results from a systematic review and meta-analysis. J Affect Disord. 2020;267:78-85.

19. Grigolon RB, Trevizol AP, Gerchman F, Bambokian AD, Magee T, Mclntyre RS, et al. Is obesity a determinant of success with pharmacological treatment for depression? A systematic review, meta-analysis and meta-regression. J Affect Disord. 2021;287:54-68.

20. Anuwatmatee S, Allison MA, Shlipak MG, McClelland RL, Kramer H, Tang S, et al. Relationship of fibroblast growth factor 21 with kidney function and albuminuria: multi-ethnic study of atherosclerosis. Nephrol Dial Transpl. 2019;34:1009-16.

21. Ong KL, McClelland RL, Allison MA, Kokkinos J, Wu BJ, Barter PJ, et al. Association of elevated circulating fibroblast growth factor 21 levels with prevalent and incident metabolic syndrome: the Multi-Ethnic Study of Atherosclerosis. Atherosclerosis. 2019;281:200-6.

\section{ACKNOWLEDGEMENTS}

We thank Steven Kliewer, $\mathrm{PhD}$, for his input on the manuscript and the participants in the EMBARC study.

\section{AUTHOR CONTRIBUTIONS}

MHT designed the study, coordinated the data and sample collection, and initiated the collaboration with the Kliewer lab at UT Southwestern. MHT and BLM posed the research questions. All the authors contributed to the manuscript. AM completed statistical analysis, contributed to interpretation of the results, and wrote the manuscript. AHC, MKJ, BSG, and TLM provided intellectual input and review of the manuscript. MHT was the principal investigator on the EMBARC study and contributed to the trial design. All authors critically reviewed the manuscript.

\section{FUNDING}

The EMBARC study was supported by the National Institute of Mental Health of the National Institutes of Health under award numbers U01MH092221 (MHT) and U01MH092250 (McGrath, PJ, Parsey, RV, Weissman, MM). The content is solely the responsibility of the authors and does not necessarily represent the official views of the National Institutes of Health. The work was further supported by the EMBARC National Coordinating Center at UT Southwestern Medical Center, MHT, MD, Coordinating PI, and the Data Center at Columbia and Stony Brook Universities. Additional funds for this report were generously provided by The Hersh Foundation and Evelyn Rose.

\section{COMPETING INTERESTS}

BLM has no conflict of interest to declare. AHC has received contracted research support from Janssen Research \& Development, LLC. MHT has received funding from Agency for Healthcare Research and Quality (AHRQ), Cyberonics, Inc., National Alliance for Research in Schizophrenia and Depression, National Institute of Mental Health (NIMH), National Institute on Drug Abuse (NIDA), National Institute of Diabetes and Digestive and Kidney Diseases (NIDDK), and Johnson \& Johnson. He has also served as an advisor or consultant for Abbott Laboratories, Inc., Akzo (Organon Pharmaceuticals, Inc.), Allergan Sales LLC, Alkermes, Arcadia Pharmaceuticals, Inc., AstraZeneca, Axon Advisors, Brintellix, Bristol-Myers Squibb Company, Cephalon, Inc., Cerecor, Eli Lilly \& Company, Evotec, Fabre Kramer Pharmaceuticals, Inc., Forest Pharmaceuticals, GlaxoSmithKline, Global Medical Education, Inc., Health Research Associates, Johnson \& Johnson, Lundbeck, MedAvante, Medscape, Medtronic, Merck, Mitsubishi Tanabe Pharma Development America, Inc., MSI Methylation Sciences, Inc., Nestle Health Science-PamLab, Inc., Naurex, Neuronetics, One Carbon Therapeutics, Ltd, Otsuka Pharmaceuticals, Pamlab, Parke-Davis Pharmaceuticals, Inc., Pfizer, Inc., PgxHealth, Phoenix Marketing Solutions, Rexahn Pharmaceuticals, Ridge Diagnostics, Roche Products, Ltd, Sepracor, SHIRE Development, Sierra, SK Life and Science, Sunovion, Takeda, Tal Medical/Puretech Venture, Targacept, Transcept, VantagePoint, Vivus, and Wyeth-Ayerst Laboratories.

\section{ADDITIONAL INFORMATION}

Correspondence and requests for materials should be addressed to Madhukar $\mathrm{H}$. Trivedi.

Reprints and permission information is available at http://www.nature.com/ reprints

Publisher's note Springer Nature remains neutral with regard to jurisdictional claims in published maps and institutional affiliations.

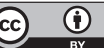

Open Access This article is licensed under a Creative Commons Attribution 4.0 International License, which permits use, sharing, adaptation, distribution and reproduction in any medium or format, as long as you give appropriate credit to the original author(s) and the source, provide a link to the Creative Commons license, and indicate if changes were made. The images or other third party material in this article are included in the article's Creative Commons license, unless indicated otherwise in a credit line to the material. If material is not included in the article's Creative Commons license and your intended use is not permitted by statutory regulation or exceeds the permitted use, you will need to obtain permission directly from the copyright holder. To view a copy of this license, visit http://creativecommons. org/licenses/by/4.0/.

(c) The Author(s) 2022 